\title{
Einführung von eLearning am Fachbereich Wirtschaft, Verwaltung und Recht - Konsequenzen und Ausblick
}

\author{
Margit Scholl
}

\begin{abstract}
Der Beitrag fasst die bisherigen Aktivitäten und Ergebnisse im Bereich eLearning am FB WVR zusammen. Er skizziert Erfahrungen aus dem internen Projekt „Lernen über Internet“, das vom 1. Juni 2004 bis zum 30. November 2004 erfolgreich durchgeführt wurde, und beschreibt erste Ergebnisse des Einsatzes von eLearning-Kursen während des Wintersemesters 2004/2005. Es werden sowohl Durchführungsaspekte und grundlegenden didaktischen Elemente beschrieben als auch anhand von aufgetretenen Problemen Grundfragen multimedialen Lehrens und Lernens behandelt.

Basierend auf spezifische Anforderungen an ein eLearning-System für den FB WVR, die u.a. für alle Rollen der Nutzung neben einfachem, intuitivem Erfassen auch klare, übersichtliche Strukturen und eine didaktische Ausrichtung beinhalten, kam die SuDiLe-Lernplattform zum Einsatz. Sie hilft gerade nicht-technischen Autoren bei der schnellen Erstellung von didaktisch ausgerichteten Inhalten.

Mit einer konsequenteren Blended-Learning-Struktur, intensiveren tutoriellen Betreuung und elektronischen Anfangs- und Zwischentests soll zukünftig versucht werden, selbstorganisiertes Lernen und begleitendes eLernen mit Hilfe der SuDiLe-Lernplattform am FB WVR zu festigen. Dies würde auch dem aktuellen Umstellungsprozess auf Bachelor- und Masterstudiengängen entsprechen.
\end{abstract}

\section{Hintergrund}

Der Bedarf nach lebensbegleitenden, zeit- und ortsunabhängigem Lernen steigt in allen Bereichen unserer „Wissensgesellschaft" stetig an, so dass elektronisches Lernen (eLearning) trotz aller bekannten Probleme weiterhin an Bedeutung gewinnen wird. Auch aus Sicht der zukünftigen Hochschulausbildung, die in Form von Bachelor-/ Masterstudiengängen zunehmend eigenverantwortliches Lernen der Studierenden unter modernen Bedingungen im Visier hat, ist es schon jetzt notwendig, eigene Erfahrungen im Einsatz von Lernplattformen und Nutzerakzeptanz zu sammeln und auszuwerten. Die Anforderungen an solche eLearning-Systeme (siehe [1]) betreffen nicht nur die Nutzerakzeptanz von Lernenden, sondern beziehen einfache Handhabung ebenso auf die Ersteller von Inhalten (Autoren) wie auf die Betreuer der Kurse (Tutoren) und die Manager der Lernplattform (Administratoren). Darüber hinaus sollte ein didaktisches Konzept so integriert sein, dass es die Nutzer in allen Rollen unterstützt, die Lernplattform intuitiv und einfach erfahren zu können. Ebenso sollten inzwischen übliche elektronische Kommunikationsformen wie eMail, Chat und Forum vorhanden sein sowie die Möglichkeit eines kooperativen und collaboraten Arbeitens (gemeinsamer Austauschbereich, Dateienstruktur, Up- und Download, Kalender) ermöglicht werden.

Im Mai letzten Jahres wurde auf Anregung des studentischen Fachschaftsrates und mit Unterstützung des Präsidenten von der Autorin das interne Projekt „Lernen über Internet“ mit dem Ziel ins Leben gerufen, die zukunftsweisende Verbindung zwischen Informationstechnik und rechtlichen Inhalten für Studierende des Fachbereichs Wirtschaft, Verwaltung und Recht (FB WVR) praktisch erfahrbar zu machen. Genutzt wurde dazu die SuDiLe-Lernplattform, die nach erziehungswissenschaftlich-psychologischen Gesichtspunkten von der Sudile - durchdachtes Lernen GbR ${ }^{1}$ entwickelt worden ist, in der IT-Weiterbildung mit Erfolg eingesetzt wird ${ }^{2}$ und deren didaktisches Konzept sich mit den Erkenntnissen deckt, die die Autorin in ihren Forschungsuntersuchungen [1] aufgezeigt hat, so dass die SuDiLe-Lernplattform die genannten Anforderungen umfassend erfüllt. SuDiLe steht für Supported Distance Learning. Die Autorin verfolgte mit dem Einsatz dieser Lernplattform letztlich das Ziel, eLearning mit lerntheoretisch fundiertem Hintergrund speziell für nicht-technisch orientierte Klientel und insbesondere für nicht-technische, z. B. juristische Inhalte gemeinsam mit Kolleginnen und Kollegen für die Studierenden des Fachbereichs möglichst zeitnah aufbauen zu können und zugänglich zu machen. Als Unterziele würden u.a. sich ein Einsatz solcher eLearning-Kurse auch für die Fernstudiengänge oder für Weiterbildungsmaßnahmen ergeben können und weitere Kooperations- und Drittmittelprojekte möglich werden, so dass die Attraktivität des FB WVR und insgesamt der TFH Wildau steigen würde.

Da die positiven Erfahrungen mit der SuDiLe-Lernplattform auf deren Einsatz in der IT-Weiterbildung basierten, sollten im ersten Schritt unseres internen Projekts der Umgang speziell innerhalb der Informatikausbildung der nicht-technischen und jahrgangsstarken Präsenzstudiengänge des FB WVR in vielfältiger Weise vorgenommen werden. Es konnte allerdings davon ausgegangen werden, dass unsere konkreten Rahmenbedingungen deutlich schwieriger im Vergleich zu isolierten PC-Weiterbildungsveranstaltungen sind. Daher sollte der Einsatz verschiedenartiger "SuDiLe-Kurse“ in unterschiedlichen Studiengruppen (Verwaltung und Recht, Wirtschaft und Recht, Europäisches Management) auf Akzeptanz und Lernmöglichkeiten hin untersucht werden. Gleichzeitig sollte bereits der Aufbau von juristischen Kursen getestet werden, um konkretere Akzeptanzhinweise für eLearning seitens nicht-technisch orientierter Autoren zu erhalten. Die beiden Studenten Serdar Coskun und Görhan Yigitbas stellten sich als Autoren für die Erstellung zweier juristi- 
scher Kurse zur Verfügung und wurden vom Kollegen RD Gerhard Guldner juristisch betreut. Darüber hinaus sollten im Projekt generelle Erfahrungen mit dem Aufbau von Kursen und der Einbeziehung von existierenden Materialien gesammelt werden.

Eine Einführung von eLearning am FB WVR ist kein einfaches Unterfangen, herrscht doch im Kreis der juristischen Fachkollegen (keineswegs nur an unserer Hochschule) meist die Haltung einer Technikskepsis vor, die erhebliche und standhafte Vorbehalte gegenüber elektronischem Lernen hervorbringt sowie auf die Grenzen des elektronischen Verfahrens verweist. In der Anfangsphase eines solchen Projektes sollte daher das Erarbeiten der theoretischen Grundlagen in den Vordergrund stehen und dessen Einsatz erprobt werden. Erst nach einer weiteren dokumentierten Bewährung sollte an differenziertere Schritte wie z.B. unterstützende Fallstudien gedacht und eine generelle Ausweitungen angestrebt werden. Es sind somit verschiedene Stufen zu überwinden, doch war und ist das begonnene interne Projekt „Lernen über Internet“ die konkrete Chance, diesen Prozess gezielt einleiten zu können. Über das Projekt, die SuDiLe-Lernplattform, die Idee ihres Einsatzes an der TFH Wildau und über verschiedene Aspekte der Durchführung von der Anfangsphase bis heute konnte an unterschiedlicher Stelle berichtet werden [2-7]; Präsentationsfolien sind der Website ${ }^{3}$ der Autorin zu entnehmen. In diesem Beitrag werden die wichtigsten Aspekte unter Verzicht auf Abbildungen zusammengefasst und der aktuelle Fortgang dargestellt. Der eingerichtete Webzugang der eLearning-Plattform hat die Adresse http: //www.sudile.com/tfh-wildau/.

\section{Phase 1: Das interne Projekt „Lernen über Internet" Juni bis November 2004}

Über den oben genannten, seit Juni 2004 eingerichteten Webzugang für die TFH Wildau erhält jeder legitimierte Nutzer entsprechend seiner Rolle einen individuellen Zugang zur SuDiLe-Lernplattform. Von dort aus kann auf die Lerninhalte und die Kommunikationsinstrumente zugegriffen werden. Das Design der Plattform ist den Rollen angepasst und mit entsprechend unterschiedlichen Funktionen versehen. Gerade die Design-Ziele standen bei der Entwicklung der SuDiLe-Lernplattform im Vordergrund. Besonders ist herauszuheben, dass die Plattform nicht als Erweiterung bestehender Produkte konzipiert wurde, sondern von Anfang an als Spezialanwendung für die Umsetzung didaktischer Konzepte im elektronischen Lernen von Sudile GbR entwickelt wurde.

Das einfache Erstellen von Lerninhalten ist - neben der generellen didaktischen Integration von offenen Seminarmethoden - eine entscheidende Innovation der Sudile-Lernplattform. Darüber hinaus wird bei der Entwicklung der SuDiLe-Lernplattform auf den Einsatz zukunftsweisender Technologien geachtet. Alle Daten werden im XML-Format auf dem Server vorgehalten, wobei sich der Zope-Server der Firma Zope Cooperation ${ }^{4}$ als geeignet erwiesen hat. Auf technische Ausführungen soll an dieser Stelle verzichtet werden, doch sei angemerkt, dass die Daten in einer Objekt-Datenbank (ZODB) gehalten und die Inhalte mit der objektorientierten Programmiersprache Python ${ }^{5}$ in der Datenbank bearbeitet werden. Als besonders qualitätssichernde Technik sollte auch noch die im Zope-Server verwendete Template-Technik ZPT6 (Zope Page Templates) genannt werden. Die SuDiLe-Plattform basiert auf Open Source Software, ist selbst allerdings keine solche. Da beim Einsatz der Plattform aber Open Source Software genutzt werden kann7 , fallen keine zusätzlichen Lizenzkosten an. Der Einsatz am FB WVR wurde durch die Definition des interenen Projekts „Lernen über Internet“ als gemeinsames, zeitlich befristetes Kooperationsprojekt kostengünstig möglich.

Besonders wichtig für den Einsatz am FB WVR war, dass das Rollenkonzept der SuDiLe-Lernplattform für Lernende, für Autoren, für Tutoren und für Administratoren ein intuitives Herangehen fördert. So können Fachspezialisten, die über wenige Computerkenntnisse verfügen, als Autoren gewonnen und ihre Inhalte sehr schnell eigenständig erstellen bzw. ohne HTML-Kenntnisse verändern. So kann auch der generelle administrative Aufwand gering gehalten werden. Das Rollenkonzept bedeutet:

- Lernende können sich eigenständig mit eigenem Passwort registrieren, schalten den gewählten Kurs über eine im System automatisch erzeugte TAN eigenständig frei, sehen ihre ggf. in verschiedenen Sprachen konzipierten Kurse, können u.a. über eine als Themenübersicht konzipierte MindMap auf Inhalte des Kurses zu greifen, haben pro Kurs einen „Laufzettel“ zum Festhalten ihres Lernfortschritts, können über Chat, eMail oder Forum kommunizieren, können Lösungen dem Tutor termingerecht (Kalenderfunktion) senden und dem Autor jederzeit Hinweise zur Verbesserung des Kurses senden, können private Termin- und Aufgabenverwaltung (z. B. „Wochenplan") definieren, können über die Plattform mit anderen gemeinsam arbeiten und Dateien austauschen (collaborates Lernen).

- Tutoren betreuen und kommunizieren auf entsprechenden Wegen mit den Lernenden, haben den Überblick über alle Laufzettel, alle Lösungen und alle Hinweise, können eine Registrierung über die TAN überprüfen.

- Autoren erstellen Inhalte als „Stationen“ in intuitv einfacher Art und Weise, indem sie eine zur Verfügung gestellte OpenOffice-Vorlage mit Hinweisen zu Themen, Lernzielen, Aufgaben, Datei-Download etc. eigenständig ohne HTML-Kenntnisse ausfüllen. Alle aus der Textverarbeitung gewohnten Funktionen können verwendet werden, wie das Einfügen von Grafiken, Listen und Aufzählungen, Tabellen, Formatierungen, Ausrichtungen, Verknüpfen von Dateien oder Setzen von Hyperlinks. Durch die Vorlage enthalten die Stationen einheitlich den Titel und die Information, ob die Lernstation bearbeitet werden muss oder freiwillig bearbeitet werden kann, enthalten konkrete Lernziele, die konkreten Aufgaben der Lernstation und alle Informationen, um diese Aufgaben eigenständig lösen zu können. Der Kursautor kann jederzeit solche Stationen auf dem Server hoch- oder runterladen, damit schnell verändern bzw. verbessern.

- Administratoren verwalten Kurse und Benutzer ebenfalls in einfacher Art und Weise.

Zusammengefasst werden in die SuDiLe-Lernplattform Methoden des „offenen Lernens“ übernommen.[8] Das 
didaktische Konzept beinhaltet eine Themenlandkarte als Überblick des eLearning-Kurses in Form von einer elektronischen MindMap, deren netzartige Struktur lernmotivierend wirkt.[9] Eine Mindmap wird im Uhrzeigersinn und die Unterzweige linear von oben nach unten gelesen. Doch gerade die Vernetzungsstruktur der Mindmap ermöglicht eine lernerspezifische, sprunghaft an Interessen oder Defiziten ausgerichtete Bearbeitung eines Kurses und die Verankerung vorhandenen Wissens. Die Aufgaben in Form des Stationenlernens rufen bei Lernenden Wiedererkennungseffekte hervor und werden den Autoren als didaktische Hilfen bei der Kursentwicklung zur Seite gestellt. Der Aufbau der Station wird den Autoren zwar in Form einer OpenOffice-Vorlage vorstrukturiert, kann jedoch von jedem Autor unterschiedlich genutzt werden. Ebenso lernmotivierend wirken die so genannten Laufzettel. Die Lernmotivation kann so durch die Funktionen der Lernplattform, durch die gezielte Aufbereitung des Lerninhalts, aber auch infolge der Betreuung durch die Tutoren unterstützt werden.

Die Lernplattform SuDiLe kann

- seminarbegleitend (in Präsenzseminare integriert)

- als Blended Learning (Online- und Präsenzphasen kombinieren) und/oder

- als reines eLearning (Online lernen) eingesetzt werden.

Innerhalb des internen Projekts „Lernen über Internet“ wurden die folgenden, in der allgemeinen Weiterbildung (PC- Software-Schulungen von Microsoft-Produkten) bereits eingesetzten IT-Kurse angeboten:

- Word-Grundlagen

- Excel-Grundlagen

- HTML-Grundlagen

- Projektmanagement mit Project.

Zwei weitere Kurse sollten bereits vorhandenes Material orts- und zeitunabhängig auf der Lernplattform für unsere registrierten Studierenden zugänglich machen. In diesem Fall wurde das didaktische Konzept des Stationenlernens stark reduziert, indem lediglich das vorhandene Videomaterial anhand einer MindMap abgerufen werden kann, ohne dass dieses Material als Lernstation neu konzipiert wurde. Es konnte somit getestet werden, wie schnell Vorhandenes als Kursgerüst auf der Plattform eingestellt werden kann. Es handelt sich dabei um die Kurse:

- Einführung in die Informatik ${ }^{8}$

- MindManager per Video erlernen (in Deutsch und Englisch).

Darüber hinaus fanden erste konzeptionelle Überlegungen für einen Datenbankkurs mit Access und für einen Kurs über Dokumenten- und Vorgangsmanagement mit FAVORIT-OfficeFlow ${ }^{9}$ statt. Zusätzlich wurden die beiden folgenden juristischen Kurse neu entwickelt:

- Grundlagen und Grundbegriffe des Arbeitsrechts

- Einführung: Staatsorgane.

Hier ist die Idee, über eLearning eventuelle Lücken der Studierenden im juristischen Basiswissen schnell und nach Bedarf schließen zu können. Diese beiden Grundlagenkurse vermitteln daher Kenntnisse von grundlegenden Definitionen, Skizzen und Schemata. Sie werden erst im derzeitigen Sommersemester 2005 den Studierenden (der Seminargruppe WR03) aktuell zu Testzwecken zur
Verfügung gestellt. Mit dem einführenden Kurs über die Staatsorgane soll den Studienanfängern bereits im ersten Semester die Möglichkeit gegeben werden, sich mit Hilfe von e-Learning Begrifflichkeiten eigenverantwortlich anzueignen, die für das Pflichtfach Verfassungsrecht bedeutend sind. Falls der Kurs zukünftig in Richtung Verwaltungsrecht ausgebaut werden würde, könnte er auch studienbegleitend angeboten werden. Kurse wie „Staatsorgane“ könnten an jeder Fachhochschule oder Berufsschule in der Ausbildung von Interesse sein, die kaufmännische, Wirtschafts- und Rechtsfächer während der Ausbildung unterrichten, d.h. ein übergreifender Austausch ist denkbar. Unser erster Arbeitsrecht-Kurs stellt ein grundlegendes Handwerkszeug für die Fallbearbeitung bereit, deren Kenntnis eine Voraussetzung in den weiteren Vorlesungen zu Arbeitsrecht darstellt. Er wendet sich primär an Studierende unserer Hochschule, unter anderem auch an diejenigen, die neben ihrer Berufstätigkeit am Fernstudium teilnehmen. In einem zweiten Schritt könnten die Lernbausteine jedoch auch an Personen gerichtet sein, die aufgrund Ihrer Stellung im Betrieb (u.a Betriebsratsmitglieder oder Personalleiter) begriffsnotwendig mit gleichwertigen Kenntnissen ihre allägliche Arbeit bewältigen. Aufgrund der Tatsache, dass sich Arbeitsrecht in vielen Berufszweigen wiederfindet, dürfte es generell bei Betriebs- und Volkswirten, Ingenieur- und Naturwissenschaftler sowie Geistes- und Sozialwissenschaftler auf Interesse stoßen, so dass hier auch Fort- und Weiterbildungskurse vorstellbar sind.

\section{Phase 2: Ergebnisse des Einsatzes von eLearning September 2004 bis Januar 2005}

Mit Hilfe des an der TFH Wildau durchgeführten, intern finanzierten Projektes „Lernen über Internet“ konnte in kurzer Laufzeit gezeigt werden, dass eLearning am FB WVR schnell, zielgerichtet, hierarchieübergreifend, interdisziplinär und kostengünstig eingerichtet und entwickelt werden kann.

Natürlich bedarf es einer Einführungsschulung für alle Nutzer in den für sie notwendigen Rollen. Nach unseren Erfahrungen kann die SuDiLe-Schulung für technisch orientiertes Personal sehr kurz gehalten werden (in etwa ein halber Tag). Selbst für nicht-technisch orientierte Tutoren und Autoren wie Juristen sind die Rollen und Funktionen in einer Zwei-Tage-Schulung ausreichend geklärt, doch sollte eine gewisse begleitende Unterstützung und Hilfestellung während der Entwicklung ihres Kurses zur Verfügung stehen, während technisch orientierte Autoren sehr gut mit den online zur Verfügung stehenden Ausführungen zurechtkommen. Im späteren Verlauf der Kursentwicklung sind für alle Autoren Lernberatung, didaktische Hilfen und Kursbewertungen sinnvoll. Den Studierenden ist ihre Rolle und die Registrierung innerhalb einer Unterrichtsstunde hinreichend erklärt, doch muss im weiteren Unterrichtsverlauf im Sinne eines „Blended Learning“ stärker als gedacht gemeinsames Lernen, Zeigen und Üben eingeplant werden.

Beispielsweise wurden die Studierenden des ersten Semesters anfangs anhand der sieben Module des Europäischen Führerscheins nach ihren PC-Kenntnissen und 
Fertigkeiten befragt. Ihre grobe Selbsteinschätzung schien die Einschätzung der Dozentin zu bestätigen, Textverarbeitungssoftware wie Word inzwischen voraussetzen zu können und nur noch Automatisierungstechniken für wissenschaftliches Arbeiten und Diplomarbeiten selbst zeigen zu müssen. Der Grundlagenkurs Word wurde daher den Studierenden als Instrument zur Verfügung gestellt, Defizite eigenverantwortlich überprüfen und ggf. beheben zu können. Ursprünglich sollte der Kurs den Studierenden nur vier Wochen zur Verfügung stehen, um eine „Überfrachtung" des eLearning-Angebots zu vermeiden. Doch auf Wunsch der Studierenden blieb der Zugriff während der gesamten Vorlesungszeit bestehen; vermutlich hat es jedoch bei nicht wenigen dazu geführt, das eigenverantworliche Lernen hinauszuschieben. Ab der vierten Semesterwoche wurde der zusammengestellte Kurs „Einführung in die Informatik" als Vorlesungsbegleitung freigeschaltet. Hier zeigte sich, dass die meisten Studierenden den Kurs als zu umfangreich ansahen und die Verbindung mit der Behandlung des Themas in der Vorlesung trotz Hinweise nicht umfassend herstellen konnten oder wollten. Im letzten Drittel des Semesters, ab Dezember 2004, konnten die Studierenden den Excel-Grundkurs nutzen, um sich in den unbetreuten Parallelstunden ${ }^{10}$ entsprechend ihres Vorwissens einzuarbeiten. Weitere Vorlesungs- und Übungsinhalte wurden überwiegend in traditioneller Art und Weise abgehalten.

Alle involvierten Studierenden wurden Ende November 2004 gebeten, einen im SuDiLe-System enthaltenen Fragebogen sinngemäß auszufüllen, um erste Hinweise hinsichtlich der Akzeptanz zu erhalten. ${ }^{11}$ Diese Nachfrage ergab eine hohe Akzeptanz des Systems. Persönliche Gespräche gingen in die gleiche Richtung einer guten Akzeptanz des Gesamtsystems. Die anfänglich recht positive Grundstimmung verweist darauf, dass die Lernplattform vor allem einfach zu bedienen ist sowie eine klare Struktur der Funktionen und der Lerninhalte aufweist. Wir können davon ausgehen, dass mit der Einführung der SuDiLe-Plattform im Kern die ersten beiden von den vier Ebenen der Evaluation von Kirkpatrick (siehe [10]) positiv besetzt werden:

- Etliche Lernende reagieren positiv auf das Lernangebot (1. Ebene) und

- die recht hohe Akzeptanz ist eine gute Voraussetzung für Lernerfolge (2. Ebene).

Lernprozesse können damit flexibel organisiert werden. Lernende können Zeitpunkt, Zeitdauer, Lernweg und Lernort individuell bestimmen. Lernende können in Teams arbeiten, Probleme über Kommunikationsnetzwerke und durch Kooperation mit anderen Lernenden lösen. Das ist die Idealvorstellung - weitere Fragenbeantwortungen zeigten jedoch, dass nur relativ wenige der Studierenden einem Austausch mit anderen Lernenden durchgeführt haben. Die großen Kenntnisunterschiede bei den Studierenden haben einen Austausch nicht automatisch beflügelt.

Im Zuge der generellen Lehrevaluation im Januar 2005 wurden dann folgende Kritikpunkte als wesentliche Problemfelder aufgeführt:

- Das eLearning sei sehr umfangreich und erfordere einen zu hohen Arbeits- und Zeitaufwand.
- Das eLearning gehe zu sehr ins Detail, die Anforderungen seien viel zu hoch.

- Das viele Lesen vor dem Monitor sei für Augen und Kopf sehr anstrengend.

- SuDiLe sei eine gute Idee, aber der Einsatz nur über Internet sei problematisch und kostenintensiv - es sollte die Möglichkeit zum Herunterladen der Dateien geben, damit man sie zu Hause in Ruhe ansehen kann.

Diese Kritikpunkte verweisen auf bekannte Problemfelder und führen zu der Erkenntnis, dass die Arbeit mit den SuDiLe-Kursen den Lernprozess bei noch nicht allen Studierenden wie von der Lehrkraft geplant unterstützt. Wie Tergan (2004) treffend ausführt (siehe [11]), haben sich bei eLearning neue Probleme ergeben, mit denen vorher kaum jemand gerechnet hatte, $u$. a. eine kognitive Überlastung von Lernenden durch die Vielfalt und Komplexität sowie eine vielfach noch fehlende Kompetenz, die neuen Medien effektiv für das Lernen zu nutzen. Offensichtlich müssen wir auch im Hochschulbereich eine intensivere Betreuung der Lernenden durch Tutoren und eine stärkere Integration der unterschiedlichen Elemente in Blended-LearningStrukturen aufbauen. Tergan verweist allerdings auch auf den Umstand, dass „letztlich ... Lernende durch die Art ihres Vorgehens beim Lernen das absolute Veto darüber (haben), ob und welche Informationen eines Lernangebots über Wahrnehmungsprozesse ihr Gehirn erreichen, dort entsprechend eigenen Intentionen (Lernzielen) bzw. entsprechend den Anforderungen einer Aufgabenstellung weiterverarbeitet, mit bestehenden Wissensstrukturen durch konstruktive kognitive Prozesse verknüpft und damit Bestandteil des individuellen Wissens werden". Aus Einzeläußerungen ist zu vermuten, dass Studierende von Präsenzstudiengängen eLearning eingeschränkter akzeptieren als Studierende von Fernstudiengängen - dies bleibt aber Gegenstand weiterer Untersuchungen. Es zeigt sich auch, dass die Kombination von angeleiteten und selbst gesteuerten Lernphasen konkreter am bereit gestellten Material gezeigt werden muss.

Tutoren sollten Lernende intensiv betreuen. Auch unsere bisherigen Ergebnisse zeigen, dass die Rolle von Tutoren für eine hohe Nutzerakzeptanz der Lernenden von zentraler Bedeutung ist. Den Kursen zugeordnete Tutoren sollten die Lernenden während der Online- und Präsenzphasen betreuen. Die Betreuung könnte am FB WVR über die SuDiLe-Lernplattform hinaus auch über Videokonferenz erfolgen. ${ }^{12}$ Während in Weiterbildungskursen, die in zeitlich begrenzten Blöcken und zu einem inhaltlich sehr begrenzten Thema durchgeführt werden, idealerweise die Tutoren auch die Moderatoren der Präsenzphasen sind, in denen selbstständiges Lernen mit den Lernenden schrittweise geübt werden kann, stellt sich die Situation an einer Fachhochschule anders dar. Es fehlt an Fachhochschulen i.d.R. der universitäre Mittelbau und studentische Tutoren sind nur begrenzt einsetzbar. Damit käme auf die Dozenten bei regulären 18 SWS-Lehrverpflichtung und insgesamt ca. 200 studentische Teilnehmer mit der parallelen Einführung von eLearning ein erhebliches Betreuungs- und Kapazitätsproblem hinzu. Sollen die Dozenten gar als aktive Tutoren fungieren und mehr als Beantworter von Fragen und Helfer bei Problemen sein, dann resultiert für sie bislang eine erhebliche Mehrarbeit. 


\section{Konsequenzen}

Wie Schenkel und Tergan (2004) [10] ausführen „berührt die Einführung von eLearning die Strukturen und Prozesse der Weiterbildung insgesamt. Es bedarf z. B. veränderter didaktischer Konzeptionen, um eLearning in der Aus- und Weiterbildung zu integrieren. Die Rollen von Trainern und Lernenden ändern sich. Der Zusammenhang ... ist jedoch nicht linear. Gute Lernangebote garantieren keine Lernerfolge. ... Lernen ist immer die Leistung einer Person. Deshalb ist es nie ein Medium allein, das Lernerfolge ermöglicht, sondern die aktive Auseinandersetzung des Lernenden mit dem über ein Medium angebotenen Wissen in einer bestimmten Lernumgebung". Diese generelle Lernumgebung muss erst bewußt geschaffen werden. Zentral sind hier die generellen Einstellungen aller Dozenten.

Das Lernen basiert größtenteils auf Eigenaktivitäten der Lernenden. Unterstützung sollen die Lernenden durch empfohlene Lernpfade, Hinweise oder Rückmeldungen erfahren. Nach den ersten Erfahrungen in den Direktstudiengängen des FB WVR sieht die Autorin die Verbesserung in dem Einsatz von Tutoren und in dem konsequenten Aufbau einer stärkeren Blended-Learning-Struktur, in der Präsenzzeiten und Online-Phasen des Lernens integrativer miteinander kombiniert werden. So liegt es nicht in der Struktur klassischer Präsenzseminare, individuelles Lernen zu fördern. Sie sollen zukünftig stärker zum angeleiteten Austausch mit unmittelbaren Absprachen und Diskussionen ausgebaut werden. eLearning fordert von den Lernenden die notwendigen Computerkenntnisse, eine hohe Lernmotivation sowie die Fähigkeit zum selbstständigen Lernen. Sind diese Anforderungen erfüllt, wird individuelles Lernen via Internet möglich. Die kommunikativen Möglichkeiten sind auch im eLearning vorhanden. Allerdings kann die „Face-to-face-Interaktion“ gerade bei Lernenden mit wenig Vorkenntnissen offenbar nicht ersetzt werden.

Zentral ist dabei die Betreuung durch die Tutoren, die vor Ort, per eMail, im Forum oder im Chat erfolgen kann. Die einzelnen Fähigkeiten und Kenntnisse eines Tutors sollten folgende sein: die Vertrautheit mit der Perspektive des Lernenden, das Moderieren von Online-Diskursen, das Tutoring von Online-Kursen und Kenntnisse in offenen Lernmethoden. Die Dozenten können nur dann auch als Tutoren agieren, wenn solche integrativen Unterrichtsmethoden mit anderen Anrechnungsfaktoren versehen werden. Ansonsten müssen Tutoren zusätzlich zugeordnet werden und eng mit dem Fachdozenten zusammenarbeiten, stellen quasi die Schnittstelle zwischen Dozenten und Studenten dar. Die Aufgaben sind u. a. die permanente Betreuung der Studierenden, Umsetzung der Lerninhalte und auch Organisation der reibungslosen Abläufe. Für eLearning, das integrativ den juristischen Unterricht begleiten soll, wären explizit von Tutoren angebotene und durchgeführte Übungen bzw. Tutorien in den PC-Laboren sinnvoll.

\section{Ausblick}

Neben dem erprobten Einsatz und relativ schnellen, lerntheoretisch basierten Aufbau von eLearning-Kursen auch im rechtlichen Bereich konnte das im internen Projekt „Lernen über Internet" vorhandene Know-how genutzt werden, um den interdisziplinären Ausbau über diverse Projektanträge zu formulieren. Eine Drittmittelförderung wäre natürlich wünschenswert, um entsprechende Ressourcen für eine zielgerichtete Etablierung von eLearning zur Verfügung zu haben. Denn nur wenn es gelingt, über Drittmittel die allgemeinen Rahmenbedingungen an Fachhochschulen für eLearning positiv zu verändern, stehen Kapazitäten und Ressourcen für die Entwicklung weiterer Kurse wie Arbeitsrecht, Verfassungsrecht, BGB, Europarecht oder Volkswirtschaftslehre, BWL, Controlling, Marketing zur Verfügung.

Doch auch ohne Drittmittelförderung müssen Wege der Weiterentwicklungsmöglichkeit gefunden werden, denn die mit dem internen Projekt verbundenen, vielfältigen und interdisziplinären Aspekte des gemeinsamen Forschens, Entwickelns und Arbeitens sollten gefestigt werden. Letztlich könnte es sich hinsichtlich möglicher Weiterbildungskurse sogar als Geschäftsfeld der TFH Wildau entwickeln.

Die Ausweitung des internen Projekts unter Entwicklung eines gemeinsamen Geschäftsmodells ist aktuell als neu definiertes Kooperationsprojekt geplant. Bis Ende des Jahres 2005 sollen in Absprache mit verantwortlichen Kollegen der TFH Wildau die folgenden vier eLearningKurse erstellt werden:

- Arbeitsrecht

- BGB I: Vertragsrecht

- Grundlagen Rechnungswesen I

- Modularer Datenbankkurs Access

Sollte dies tatsächlich gelingen, dann würde eine wirklich positive Lernumgebung geschaffen werden, die als lernfördernder Kontext gesehen werden kann. Gleichzeitig wird durch die enge Kooperation zwischen TFH Wildau und Sudile GbR auch die Entwicklung der SuDiLe-Lernplattform selbst vorangetrieben. So sah der Entwurf der Sudile-Lernplattform ursprünglich keine barrierefreie Nutzung als vorrangiges Ziel vor. Inzwischen ist die Barrierefreiheit erklärtes Ziel der Entwickler der Sudile GbR. Ebenso stehen die sprachliche Internationalisierung (Deutsch-EnglischPolnisch) sowie ein Test- und Zertifizierungssystem auf der Tagesordnung. Und, infolge der studentischen Kritik, wird eine Offline-Möglichkeit der Kurse geplant.

Mit einer konsequenteren Blended-Learning-Struktur, intensiveren tutoriellen Betreuung und elektronischen Anfangs- und Zwischentests könnte zukünftig versucht werden, selbstorganisiertes Lernen und begleitendes eLernen mit Hilfe der SuDiLe-Lernplattform am FB WVR zu festigen.

Darüber hinaus wird mit der aktuellen Umstellung unserer Studiengänge auf Bachelor-/Master-Studiengänge ein erhöhter Anteil an Eigenstudium manifestiert werden, der selbstorganisiertes Lernen von den Studierenden voraussetzt. Begleitendes eLearning wird die Chance bieten, diese Herausforderung zu bewältigen. Doch wie die Auflistung der heterogenen Interessen der Akteure an eLearning von Goertz und Johanning (2004) [12] zeigt, führt dies „beinahe zwangsläufig zu Zielkonflikten“. Qualität von eLearning ist nicht absolut, sondern hängt von den Zielen der Interessenten ab. Immerhin wäre der Grundstein gelegt, eLearning an unserem nicht-technischen FB WVR unter didaktischen Gesichtspunkten, auf geprüfter und bewährter Art und Weise, einfach und schnell voran zu bringen. 


\section{Anmerkungen}

1 Christian Niemczik \& Peter Koppatz, Sudile - durchdachtes Lernen GbR, Jägerstraße 36, 14467 Potsdam, eMail: info@ sudile.com, http://www.sudile.com

2 U.a. im Landesbetrieb für Datenverarbeitung und Statistik des Landes Brandenburg (LDS BB), Potsdam.

3 http://www.tfh-wildau.de/web_wvr/scholl/praesent/

4 http://www.zope.com/Products/Zope.html

5 http://www.python.org

6 http://zpt.sourceforge.net/

7 z. B. können die Autoren beim Erstellen der Inhalte das kostenlose OpenOffice nutzen; siehe http://www.openoffice.org

8 Das Videomaterial dieses Kurses war ursprünglich unter Leitung des Kollegen Prof. Dr. Hendrix von Studierenden für Studierende im FB BW/WI entwickelt worden und wurde uns freundlicherweise für das Projekt hochschulintern zur Verfügung gestellt.

9 FAVORIT®-OfficeFlow ${ }^{\circledR}$ steht für Flexibles Archivierungs- und VORgangsbearbeitungssystem im IT-gestützten Geschäftsgang und ist eine praxisbewährte Software-Lösung des Bundesverwaltungsamtes (BVA). Die TFH Wildau war die erste Hochschule, die das komplexe Softwaresystem in die Lehre im Jahre 2001eingeführt hat und seit 2002 in der Informatikausbildung des Studiengangs Verwaltung und Recht nutzt. Unser erster Erfahrungsbericht ist nach wie vor der folgenden Webseite des BVA zu entnehmen. http://www.bva.bund.de/favorit/kunden/ 00022/index.html

10 Die Teilnehmeranzahl der einzelnen Seminargruppen liegen in etwa bei 35 Studierende. Innerhalb der Informatikausbildung steht am FB WVR für praktische Arbeiten am PC ein PC-Labor für 18 Teilnehmer und im Hochschulrechenzentrum zwei weitere Labore für jeweils 16 Teilnehmern zur Verfügung. D. h., für die PC-Übungen muss die jeweilige Seminargruppe auf jeden Fall geteilt werden. Jeweils eine der Teilgruppen hat praktischen PC-Unterricht mit der Dozentin, während die zweite, parallele Teilgruppe PC-Übungsaufgaben unbetreut in Eigenverantwortung durchführen muss. In der darauffolgenden Woche wird gewechselt. Das vor Jahren noch mögliche Verfahren, die beiden Teilgruppen für den praktischen Unterricht hintereinander zu legen und vom Dozenten betreut agieren zu lassen, kann inzwischen offenbar aus Gründen des curricularen Normwertes und des Budgets am FB WVR nicht mehr durchgeführt werden. Hier bietet sich somit der seminarbegleitende Einsatz von eLearning als Unterstützungselement zwingend an, sollte jedoch nach unseren Erfahrungen tutoriell betreut stattfinden.

11 Es handelt sich hierbei um freiwillige Rückmeldungen, keine Repräsentativumfrage. Die Rücklaufquote betrug insgesamt etwa $18 \%$.

12 Am FB WVR existiert seit 2004 infolge des erfolgreich abgeschlossenen Drittmittelprojekts „Customer Support for Joblearning on Demand“ ein Customer Support Studio für passgenaue Schulungen über Videokonferenz; siehe dazu http: //www.tfh-wildau.de/web_wvr/scholl/projekte/index.html .

\section{Literatur}

[1] Scholl, Margit (2004), IT-Didaktik: Selbstorganisiertes Lernen in der IT-Ausbildung und eLearning, Wissenschaftliche Beiträge der Technischen Fachhochschule Wildau, Heft 2/2004, ISSN 0940-8214.
[2] Niemczik, Christian, Peter Koppatz und Margit Scholl (2004), Die SUDILE - Lernplattform, Innovatives eLearning und „Blended Learning“ in der Lehre, Tagungsband, erste Potsdamer Multimedia Konferenz, Oktober 2004.

[3] Hendrix, Michael und Margit Scholl (2005), „,Personalisiertes eLearning' sowie ,Didaktik und inuitives Erfassen' an der TFH Wildau“, Gemeinsame Poster-Präsentation auf der Learntec 2005, Karsruhe, 15.-18.2.2005.

[4] Scholl, Margit, Serdar Coskun, Christian Niemczik (2005), Durchdachtes eLernen via Internet an der TFH Wildau, GML2 2005 - 3. Workshop „Grundfragen multimedialen Lehrens und Lernens“, Tagungsband, BTU Cottbus, 7.-9.3.2005.

[5] Scholl, Margit (2005), Innovatives eLearning mit SuDiLe zu rechtlichen Fragestellungen, Präsentation auf dem KoopA ADV, Kooperationsausschuss Automatisierte Datenverarbeitung (Bund/Länder/Kommunaler Bereich), 42. Erfahrungsaustausch, CD-ROM, Schwerin, 15.3.-16.3.2005.

[6] Scholl, Margit, Gerhard Guldner, Serkan Coskun, Gökhan Yigitbas, Chistian Niemczik und Peter Koppatz (2005), Instructional Supported Distance eLearning in the Sphere of Law Lectures, TESI 2005, Training, Education \& Simulation International, Conference Proceedings (CD-ROM), Maastricht, The Netherlands, 22.-24.3.2005.

[7] Scholl, Margit, Serdar Coskun, Christian Niemczik und Peter Koppatz (2005), Arbeitsrecht als innovatives eLearning mit SuDiLe, IRIS 2005, Internationales Rechtsinformatik Symposium, Salzburg, 24.-26.2.2005. In: Schweighofer u.a. (Hrsg.), Informationstechnik in der juristischen Realität - Aktuelle Fragen der Rechtsinformatik 2004, Schriftenreihe Rechtsinformatik Bd 10, Verlag Österreich, Wien, im Erscheinen.

[8] Hegele, Irmintraut (2002), Stationenarbeit - Ein Einstieg in den offenen Unterricht. In: J. Wiechmann (Hrsg.), Zwölf Unterrichtsmethoden, Beltz, 3. Auflage, 2002, ISBN 3-40725222-6.

[9] Scholl, Margit (2004), eLearning klein, aber fein: Effizienzsteigerungen von Brainstormingprozessen in Beratungen durch computergestütztes MindMapping, Zeitschrift Splitter des LIT Berlin, Heft 1/2004.

[10] Schenkel, Peter, und Sigmar-Olaf Tergan (2004), Qualität von E-Learning: eine Einführung. In: Tergan/Schenkel (Hrsg.), Was macht eLearning erfolgreich? Springer, 2004, ISBN 3540-20676-0.

[11] Tergan, Sigmar-Olaf (2004), Was macht Lernen erfolgreich? Die Sicht der Wissenschaft. In: Tergan/Schenkel (Hrsg.), Was macht eLearning erfolgreich? Springer, 2004, ISBN 3-54020676-0.

[12] Goertz, Lutz, und Anja Johanning (2004), Das Kunststück, alle unter einen Hut zu bringen. Zielkonflikte bei der Akzeptanz des E-Learning. In: Tergan/Schenkel (Hrsg.), Was macht eLearning erfolgreich? Springer, 2004, ISBN 3-540-20676-0.

\section{Autorin}

\section{Prof. Dr. Margit Scholl}

Technische Fachhochschule Wildau

Fachbereich Wirtschaft, Verwaltung und Recht

Tel. +49 3375 508-917, -570

mscholl@wvr.tfh-wildau.de

www.tfh-wildau.de/web_wvr/scholl 